\title{
TIME-DEPENDENT WKB EXPANSIONS FOR A CLASS OF PROPAGATORS
}

\author{
S.M. BLINDER \\ Department of Chemistry, University of Michigan, Ann Arbor, MI 48109, USA
}

Received 10 September 1985

\begin{abstract}
A time-dependent variant of the WKB method is developed for propagators which do not have the simple Feynman structure $K=f \exp (\mathrm{i} S / \hbar)$ with $f=f(t)$. The method is applied to radial propagators for $N$-dimensional isotropic oscillators. An expansion in ascending powers of $\hbar$ results in an asymptotic series, which sums to a simple form involving a Bessel function. Alternatively, a convergent series for the propagator is obtained by expansion in inverse powers of $\hbar$.
\end{abstract}

Semiclassical approximations to propagators are extensively applied in spectroscopy, scattering theory, molecular dynamics, irreversible thermodynamics and elementary-particle theory [1]. Terms in the WKB expansion involving higher powers of $\hbar$ are less often considered. In this paper, we develop a time-dependent formulation of the WKB method which can be systematically extended to arbitrary powers of $\hbar$. The method is applied to the radial components of the Feynman propagators for $N$-dimensional isotropic harmonic oscillators.

Consider accordingly an $N$-dimensional Euclidean space $\left(x_{1}, x_{2} \ldots x_{N}\right)$ with the generalized radial variable

$$
r=\left(\sum_{i=1}^{N} \chi_{i}^{2}\right)^{1 / 2} \text {. }
$$

For an $N$-dimensional isotropic harmonic oscillator, the partial-wave propagator for hyperangular momentum quantum number $L$ satisfies the radial Schrödinger equation

$$
\begin{aligned}
& {\left[\mathrm{i} \hbar \frac{\partial}{\partial t}+\frac{\hbar^{2}}{2 m}\left(\frac{1}{r N-1} \frac{\partial}{\partial r} r^{N-1} \frac{\partial}{\partial r}-\frac{L(L+N-2)}{r^{2}}\right)+\frac{1}{2} m \omega^{2} r^{2}\right] K K_{L}^{(N)}\left(r, r^{\prime}, t\right)=0} \\
& N=2,3,4 \ldots ; \quad L=0,1,2
\end{aligned}
$$

subject to the initial condition

$$
K_{L}^{(N)}\left(r, r^{\prime}, 0\right)=\delta\left(r-r^{\prime}\right) /\left(r r^{\prime}\right)^{N / 2-1 / 2}
$$

The form of the $N$-dimensional Hamiltonian is discussed in a recent acticle [2]. The cases $N=2$ and 3 are most familiar. The factor dividing the delta function in (3) arises from the weight function $r^{N-1}$ in the SturmLiouville formulation.

As shown by Feynman [3], for Hamiltonians reducible to quadratic forms in generalized coordinates and momenta, the propagator has the structure

$$
K=f \exp (\mathrm{i} S / \hbar) \text {. }
$$

Here $S$ represents the classical action, while the pre-exponential factor $f$ is a function of $t$ alone. For Hamiltonians not of the above type, including that represented in eq. (2), the simple reduction to eq. (4) is no longer guaranteed.

We propose to generalize the form of eq. (4) such that $f=f\left(r, r^{\prime}, t\right)$ a function of the coordinates as well as time. 
Substituting (4) into (2) and collecting like powers of $\hbar$, we obtain

$$
\begin{aligned}
- & {\left[S_{t}+(1 / 2 m) S_{r}^{2}+\frac{1}{2} m \omega^{2} r^{2}\right] f+\mathrm{i} \hbar\left\{f_{t}+(1 / m) S_{r} f_{r}+(1 / 2 m) S_{r r} f+[(N-1) / 2 m r] S_{r} f\right\} } \\
& +\left(\hbar^{2} / 2 m\right)\left\{f_{r r}+[(N-1) / r] f_{r}-\left[L(L+N-2) / r^{2}\right] f\right\}=0 .
\end{aligned}
$$

The segment of zerot order in $\hbar$ reduces to the Hamilton-Jacobi equation for a one-dimensional harmonic oscillator. The solution is well known $[3,4]$, viz.

$$
S\left(r, r^{\prime}, t\right)=\frac{1}{2} m \omega\left(r^{2}+r^{2}\right) \cot \omega t-m \omega r^{\prime} \csc \omega t .
$$

We next assume an expansion of $f\left(r, r^{\prime}, t\right)$ in ascending powers of $\hbar$. We write for convenience

$$
f\left(r, r^{\prime}, t\right)=\sum_{n=0}^{\infty}(\mathrm{i} \hbar / 2 m)^{n} f^{(n)}\left(r, r^{\prime}, t\right) .
$$

With the use of (6) and (7), eq. (5) is transformed to a recursive relation for the $f^{(n)}$ :

$$
\begin{aligned}
& f_{t}^{(n)}+\left(\omega r \cot \omega t-\omega r^{\prime} \csc \omega t\right) f_{r}^{(n)}+\left[\frac{1}{2} N \omega \cot \omega t-\frac{1}{2}(N-1) \omega\left(r^{\prime} / r\right) \csc \omega t\right] f^{(n)} \\
& \quad=f_{r r}^{(n-1)}+[(N-1) / r] f_{r}^{(n-1)}-\left[L(L+N-2) / r^{2}\right] f^{(n-1)} .
\end{aligned}
$$

As an etude which illustrates the essential features of the method in a much simplified computation, the reader might consider the free particle limit $\omega=0$ and perhaps, in addition, the "S-wave" case $L=0$.

For $n=0$ in eq. (8), an obvious trial solution of the form $f^{(0)}=\left(r r^{\prime}\right)^{\alpha}(\omega \csc \omega t)^{\beta}$ works with $\alpha=1 / 2-N / 2$, $\beta=1 / 2$. Thus

$$
f^{(0)}\left(r, r^{\prime}, t\right)=\text { const. }\left(r^{\prime}\right)^{1 / 2-N / 2}(\omega \csc \omega t)^{1 / 2} .
$$

It is readily shown that successive functions $f^{(n)}$ differ by factors proportional to $1 / \omega r r^{\prime} \csc \omega t$. We write accordingly

$$
f^{(n)}=c_{n}\left(\omega r r^{\prime} \csc \omega t\right)^{-n} f^{(0)}=\text { const. } c_{n}\left(r r^{\prime}\right)^{1 / 2-N / 2-n}(\omega \csc \omega t)^{1 / 2-n} .
$$

Substitution of (10) into (8) leads, after somewhat lengthy algebra, to the following ratio of coefficients:

$$
c_{n} / c_{n-1}=(n+L+N / 2-3 / 2)(n-L-N / 2+1 / 2) / n \text {. }
$$

For even $N$ the series (7) diverges since $c_{n} / c_{n-1} \sim n$ as $n \rightarrow \infty$. For odd $N$, the series terminates at $n=L+N / 2-1 / 2$ (or $n=3 / 2-L-N / 2$ ). In either case, $f\left(r, r^{\prime}, t\right)$ can be represented in terms of a generalized hypergeometric series:

$$
f\left(r, r^{\prime}, t\right)=f^{0}\left(r, r^{\prime}, t\right){ }_{2} F_{0}\left[(L+N / 2-1 / 2),(-L-N / 2+3 / 2) ; ; \mathrm{i} h / 2 m \omega r r^{\prime} \csc \omega t\right],
$$

where [5]

$$
{ }_{2} F_{0}(\alpha, \beta ; ; z) \equiv \sum_{n=0}^{\infty} \frac{(\alpha)_{n}(\beta)_{n}}{n !} z^{n}, \quad(\alpha)_{n} \equiv \alpha(\alpha+1) \ldots(\alpha+n-1), \quad(\alpha)_{0}=1
$$

Now the function (13) appears in the asymptotic expansion for a confluent hypergeometric function, $M(a, b, z)$. Specifically [6],

$$
M(a, b, z) \sim[\Gamma(b) / \Gamma(a)] \mathrm{e}^{z} z^{a-b}{ }_{2} F_{0}(b-a, 1-a ; ; 1 / z) \text { as }|z| \rightarrow \infty,
$$

where

$$
M(a, b, z) \equiv{ }_{1} F_{1}(a ; b ; z) \equiv \sum_{n=0}^{\infty} \frac{(a)_{n}}{(b)_{n}} \frac{z^{n}}{n !} .
$$


It is easy to show that both sides of eq. (14) satisfy Kummer's differential equation

$$
z f^{\prime \prime}(z)+(b-z) f^{\prime}(z)-a f(z)=0 .
$$

With use of (14) and (9) in (12) we obtain

$$
\begin{aligned}
& f\left(r, r^{\prime}, t\right)=\text { const. }\left(r r^{\prime}\right)^{L}(\omega \csc \omega t)^{L+N / 2} \exp \left(2 \mathrm{i} m \omega r r^{\prime} \csc \omega t / \hbar\right) \\
& \quad \times M\left(L+N / 2-1 / 2,2 L+N-1,-2 \mathrm{i} m \omega r r^{\prime} \csc \omega t / \hbar\right) .
\end{aligned}
$$

Note that $b=2 a$ in $M$, in which case reduction to a Bessel function occurs in accordance with ref. [7]

$$
J_{\nu}(z)=\frac{(z / 2)^{\nu} \mathrm{e}^{ \pm \mathrm{i} z}}{\Gamma(\nu+1)} M\left(\nu+\frac{1}{2}, 2 \nu+1, \mp 2 \mathrm{i} z\right) .
$$

Finally, substitution of (17) and (6) into (4) gives the desired radial propagator:

$$
\begin{aligned}
& K_{L}^{(N)}\left(r, r^{\prime}, t\right)=(-\mathrm{i})^{L+N / 2}\left(r^{\prime}\right)^{1-N / 2}(m \omega \csc \omega t / \hbar) \exp \left[\mathrm{i} m \omega\left(r^{2}+r^{\prime 2}\right) \cot \omega t / 2 \hbar\right] \\
& \quad \times J_{L+N / 2-1}\left(m \omega r r^{\prime} \csc \omega t / \hbar\right),
\end{aligned}
$$

in which the constant has been adjusted to fulfill the initial condition (3). The result (14) agrees with our earlier derivation based on the Fourier transform of the corresponding Green's function [2]. We note that, as is the case in the conventional WKB method, expansion in ascending powers of $\hbar$ produces an asymptotic series [8].

The propagator (19) can alternatively be derived from a convergent expansion in descending powers of $\hbar$. The calculation can be based on eq. (8) as before but now we write

$$
f\left(r, r^{\prime}, t\right)=\sum_{n=0}^{\infty}(\mathrm{i} \hbar / 2 m)^{-n} f^{(-n)}\left(r, r^{\prime}, t\right) .
$$

with $n=1$, eq. (8) admits of the solution $f^{(0)}=r^{L} \times\left(\right.$ function of $r^{\prime}$ and $\left.t\right)$. More specifically, it can be conjectured that

$$
f^{0}\left(r, r^{\prime}, t\right)=\text { const. }\left(r r^{\prime}\right)^{L}(\omega \csc \omega t)^{\beta},
$$

with $\beta$ to be determined. In analogy with $(10)$, we write

$$
f^{(-n)}=\text { const. } c_{-n}\left(r r^{\prime}\right)^{L+n}(\omega \csc \omega t)^{\beta+n} .
$$

Substitution into (8) identifies $\beta=L+N / 2$ and leads to the recursion formula

$$
c_{-(n+1)} / c_{-n}=(n+L+N / 2-1 / 2) /(n+1)(n+2 L+N-1) \text {. }
$$

This corresponds to the convergent series for the function

$$
f=f^{0} M\left(L+N / 2-1 / 2,2 L+N-1,2 \mathrm{i} m \omega r r^{\prime} \csc \omega t / \hbar\right) .
$$

With use of (18), we again arrive at the propagator (19).

For odd dimension $N$, the propagators can be expressed in terms of spherical Bessel functions. Specifically, for $N=3$, with $L=l$ :

$$
K_{l}\left(r, r^{\prime}, t\right)=(-\mathrm{i})^{l+3 / 2}(2 / \pi)^{1 / 2}(m \omega \csc \omega t / \hbar)^{3 / 2} \exp \left[\mathrm{i} m \omega\left(r^{2}+r^{\prime 2}\right) \cot \omega t / 2 \hbar\right] J_{l}\left(m \omega r^{\prime} \csc \omega t / \hbar\right) .
$$

With use of the addition theorem [9]

$$
\exp (\mathrm{i} z \cos \theta)=\sum_{l=0}^{\infty}(2 l+1) \mathrm{i}^{l} J_{l}(z) P_{l}(\cos \theta)
$$


the sum over partial waves

$$
K\left(r, r^{\prime}, t\right)=\sum_{l=0}^{\infty}[(2 l+1) / 4 \pi] P_{l}(\cos \theta) K_{l}\left(r, r^{\prime}, t\right)
$$

results in the familiar three-dimensional harmonic-oscillator propagator $[3,4]$

$$
K\left(r, r^{\prime}, t\right)=(m \omega \csc \omega t / 2 \pi \mathrm{i} \hbar)^{3 / 2} \exp \left\{(\mathrm{i} m \omega / 2 \hbar)\left[\left(r^{2}+r^{\prime 2}\right) \cot \omega t-2 r \cdot r^{\prime} \csc \omega t\right]\right\} .
$$

This propagator has, of course, the simple Feynman structure (4) with $f=f(t)$.

In a separate publication, we apply the time-dependent WKB expansion developed here to the Coulomb propagator, for which the semi-classical approximation was recently given [10].

\section{References}

[1] M.S. Child, ed, Semiclassical methods in molecular scattering and spectroscopy (Reidel, Dordrecht, 1980).

[2] S.M. Blinder, J. Math. Phys. 25 (1984) 905.

[3] R.P. Feynman, Rev. Mod. Phys. 20 (1948) 367;

R.P. Feynman and A.R. Hibbs, Quantum mechanics and path integrals (MoGraw-Hill, New York, 1965).

[4] S.M. Blinder, Foundation of quantum dynamics (Academic Press, New York, 1974).

[5] L.J. Slater, Confluent hypergeometric functions (Cambridge Univ. Press, Cambridge, 1960).

[6] M. Abramowitz and I.A. Stegum, eds., Handbook of mathematical functions (Natl Bur. Std., Washington, 1972) p. 508, eq. (13.5.1).

[7] M. Abramowitz and I.A. Stegun, eds., Handbook of mathematical functions (Natl. Bur. Std., Washington, 1972) p. 362, eq. (9.1.69).

[8] F.W.J. Olver, Asymptotics and special functions (Academic Press, New York, 1974).

[9] M. Abramowitz and I.A. Stegun, eds., Handbook of mathematical functions (Natl. Bur. Std., Washington, 1972) p. 440, eq. (10.1.47).

[10] S.M. Blinder, Phys. Rev. Letters 52 (1984) 1771. 